Article

\title{
Reduction of NF- $\kappa$ B Signals in Platelets and Prolongation of Platelet Plug Formation against High Shear Flow in Whole Blood on Human Subject by Columbianadin
}

\author{
Chih-Wei Hsia 1,+(D), Chih-Hao Yang 1,2,+ , Joen-Rong Sheu 1,2,+(D), Chih-Hsuan Hsia ${ }^{1,3}$, \\ Cheng-Lin Tsai ${ }^{4}$ (D), Wei-Chieh Huang ${ }^{1}$, Ting-Yu Chen ${ }^{2}$, Thanasekaran Jayakumar ${ }^{1}$, \\ Periyakali Saravana Bhavan ${ }^{5}$ (D) and Yi Chang $1,6,7, *$ \\ 1 Graduate Institute of Medical Sciences, College of Medicine, Taipei Medical University, Taipei 110, Taiwan; \\ d119106003@tmu.edu.tw (C.-W.H.); chyang@tmu.edu.tw (C.-H.Y.); sheujr@tmu.edu.tw (J.-R.S.); \\ T014913@ms.skh.org.tw (C.-H.H.); m120107013@tmu.edu.tw (W.-C.H.); jayakumar@tmu.edu.tw (T.J.) \\ 2 Department of Pharmacology, School of Medicine, College of Medicine, Taipei Medical University, \\ Taipei 110, Taiwan; y0513260323@tmu.edu.tw \\ 3 Translational Medicine Center, Shin Kong Wu Ho-Su Memorial Hospital, Taipei 111, Taiwan \\ 4 Graduate Institute of Metabolism and Obesity Sciences, College of Nutrition, Taipei Medical University, \\ Taipei 110, Taiwan; ma48106001@tmu.edu.tw \\ 5 Department of Zoology, Bharathiar University, Coimbatore, Tamil Nadu 641046, India; bhavan@buc.edu.in \\ 6 Department of Anesthesiology, Shin Kong Wu Ho-Su Memorial Hospital, Taipei 111, Taiwan \\ 7 School of Medicine, Fu Jen Catholic University, New Taipei City 24205, Taiwan \\ * Correspondence: m004003@ms.skh.org.tw; Tel.: +886-98-416-0920; Fax: +886-2-28-32-6912 \\ + These authors contributed equally to this work.
}

Received: 5 August 2020; Accepted: 16 October 2020; Published: 19 October 2020

\begin{abstract}
Myocardial infarction and cerebral ischemic stroke during the process of arterial thrombosis are prominently causes of death worldwide. Platelets are anucleated cells and play a critical factor in these diseases. Columbianadin (CBN), a coumarin derivative from plants, inhibits effective platelet activation. In this study, platelet function analysis revealed that the closure time of the platelet plug in human whole blood significantly prolonged by CBN, whereas CBN did not pointedly prolong the bleeding time in mice. BAY11-7082 (an inhibitor of IKB kinase) and MG-132 (an inhibitor of proteasome) inhibited collagen-stimulated platelet aggregation and ATP-release in human platelets, BAY11-7082 exhibited a higher potency than MG-132. Moreover, CBN markedly reduced NF- $\mathrm{kB}$ activation (e.g., I $\kappa \mathrm{B} \alpha$ and $\mathrm{p} 65$ phosphorylation) and reversed I $\mathrm{\kappa} B \alpha$ degradation in activated platelets. We investigated intercellular signaling events between mitogen-activated protein kinases and NF- $\mathrm{kB}$, and found that BAY11-7082 abolished JNK1/2 and ERK1/2 phosphorylation. Interestingly, SP600125 (an inhibitor of JNK) but not PD98059 (an inhibitor of ERK) had no effect in NF-KB activation in activated platelets. Moreover, CBN but not BAY11-7082 significantly reduced hydroxyl radical (HO•) formation in platelets. Therefore, we propose that CBN inhibits NF- $\mathrm{kB}$ activation in human platelets and could present a potent clinical treatment for thromboembolic diseases.
\end{abstract}

Keywords: columbianadin; human platelets; hydroxyl radical; ERK1/2; JNK1/2; NF-KB; arterial thrombosis

\section{Introduction}

The world major causes of death considered are arterial thrombotic events, such as myocardial infarction, ischemic stroke, and venous thromboembolisms [1]. Platelets are derived from megakaryocytes, 
which are small and discoid in shape and have a lifespan of 7-10 days [2]. Over activation of platelets is associated with atherothrombotic process and profoundly contribute to the pathology of venous thrombosis [3]. Platelets are adaptable fragments of cytoplasm that have the primary function of arresting bleeding. Understanding the causes of platelet activation and aggregation is imperative. Platelets are key regulators of hemostasis and thrombosis. Platelet activation is triggered by collagen along with thrombin and ADP, which are produced after the atherosclerotic vascular lesion occurred.

The NF-kB signaling pathway plays a major controller of various cellular actions, including neurodegenerative, inflammatory, and vascular pathological responses [4]. NF- $\mathrm{kB}$ translocate from cytosol into nucleus upon activation for the events of regulating gene expression. In a normal state, NF- $\kappa B$ is attached with its inhibitor of $\kappa B(I \kappa B)$ and inactively reserved in the cytosol. I $\kappa B \alpha$ is the

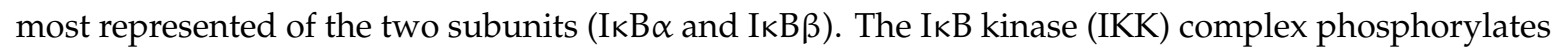
I $\kappa B$ upon activation, initiating proteasome-mediated degradation and freeing NF- $\kappa B$ for nuclear translocation [5]. Among IKK subunits $(\alpha, \beta$, and $\gamma)$, IKK $\beta$ is the most active. The role of NF- $\mathrm{KB}$ in nucleated cells is well documented [6]. Therefore, the actions of NF- $\mathrm{KB}$ have been mostly attributed to various nuclear events. Furthermore, three IKK family members are also expressed in platelets, in which $\beta$ form being more strongly expressed than $\alpha$ or $\gamma$ form $[7,8]$. NF- $\kappa B$ signaling in platelets involves IKK $\beta$ phosphorylation, IKB $\alpha$ degradation, and p65 phosphorylation [8]. However, unlike other cells, the crowning events of NF- $\mathrm{kB}$ signaling in platelets remain partially understood. For this purpose, several studies were performed utilizing either pharmacological inhibitors of NF- $\mathrm{KB}$ such as BAY 11-7082 or knockout mice to unravel the role of NF- $\mathrm{KB}$ in platelets, specifically in the context of their activation, and aggregation.

Columbianadin (CBN) is a natural coumarin-type compound that is widely used to treat spasms, rheumatisms, and headaches in Chinese medicine [9]. CBN has attracted extensive attention because of its biological activities, such as inhibition of carcinomas (e.g., colon cancer, bladder carcinoma, and human leukemia) [8], and diminished nitric oxide (NO) production by downregulating inducible NO synthase [9]. Moreover, oral administration of CBN $(20-60 \mathrm{mg} / \mathrm{kg})$ exhibited inhibitory activity against lung inflammation induced by LPS in mice [10,11].

CBN exhibited potent activity against ADP-stimulated rat platelets [5]. Hou et al. [12] reported that CBN modulates platelet activation by interfering with the integrin $\alpha_{\text {IIb }} \beta_{3}$ inside-out but not outside-in signals in human platelets. CBN was found to inhibit the phosphorylation of c-Jun N-terminal kinase (JNK) $1 / 2$ and extracellular signal-regulated kinase (ERK) 1/2. Interestingly, CBN did not inhibit p38 mitogen-activated protein kinase (MAPK) in collagen-activated platelets [12]. That study also demonstrated that CBN had remarkable activity against platelet activation through inhibition of the upstream platelet signaling pathways, such as phospholipase $C \gamma 2$, protein kinase $C$, Akt. In that, we also noticed that CBN markedly hindered integrin $\alpha_{\mathrm{IIb}} \beta_{3}$ activation by interfering with the binding of PAC-1 (FITC-anti-human CD41/CD61) [12]. Platelets are anucleated cells, they do not differentiate or proliferate and thus provide a favorable model for studying the nongenomic functions of NF- $\mathrm{kB}$. In the current study, we further evaluated the activity of CBN in prolonging the platelet plug formation under high shear flow in whole blood. We also conducted an investigation exploring the roles and relationships of NF- $\mathrm{kB}$ with MAPKs in CBN-mediated antiplatelet activity in human platelets.

\section{Materials and Methods}

\subsection{Materials}

CBN (>98\%) and MG-132 were purchased from ChemFaces Biochem. (Wuhan, Hubei, China) and Cayman Chem. (Ann Arbor, MI, USA), respectively. Dimethyl sulfoxide (DMSO), collagen (type I), luciferin/luciferase, PD98059, SB203580, SP600125, bovine serum albumin (BSA), prostaglandin $\mathrm{E}_{1}$ ( $\left.\mathrm{PGE}_{1}\right),(\mathrm{E})-3-(4-m e t h y l p h e n y l s u l f o n y l)-2-p r o p e n e n i t r i l e ~(B A Y 11-7082)$, heparin, aspirin and 5,5-dimethyl-1 pyrroline N-oxide (DMPO) were purchased from Sigma (St. Louis, MO, USA). The anti-phospho-p38 MAPK Ser ${ }^{182}$ polyclonal antibody (pAb), anti-IкB $\alpha$ (44D4), anti-phospho-IкB $\alpha$ 
$\left(\mathrm{Ser}^{32}{ }^{36}\right)(5 \mathrm{~A} 5)$, anti-phospho-c-JNK ( $\mathrm{Thr}^{183} / \mathrm{Tyr}^{185}$ ), anti-p38 MAPK and anti-NF-kB p65 monoclonal antibody $(\mathrm{mAb})$, as well as anti-phospho-p44/p42 ERK $\left(\mathrm{Thr}^{202} / \mathrm{Tyr}^{204}\right)$ and anti-phospho-NF- $\mathrm{kB}$ p65 $\left(S^{5}{ }^{536}\right)$ pAb, were purchased from Cell Signaling (Beverly, MA, USA). The anti- $\alpha$-tubulin mAb was purchased from NeoMarkers (Fremont, CA, USA). CBN was dissolved in 0.1\% DMSO.

\subsection{Platelet Function Analysis in Human Whole Blood}

The Dade Behring PFA-100 System (Marburg, Germany) was used to analyze platelet function [13]. Cartridges containing collagen/ADP (CADP)-coated membranes were preincubated with CBN (45 and $90 \mu \mathrm{M})$ or the solvent control ( $0.1 \%$ DMSO) for $2 \mathrm{~min}$. Whole blood aliquots ( $0.8 \mathrm{~mL} /$ cartridge) were applied to the cartridges before contents were exposed to high shear flow conditions (5000-6000/s). The closure time (CT) was defined as time required for a platelet plug to occlude the aperture in the collagen membrane [13].

\subsection{Tail Bleeding Time}

BioLasco, Taipei, Taiwan supplied male ICR mice (20-25 g, 5-6 weeks). Affidavit of Animal Use Protocol, Taipei Medical University (LAC-2018-0360) approved all procedures and protocols. The bleeding time was measured after $30 \mathrm{~min}$ of intraperitoneal administration of CBN (10 and $20 \mathrm{mg} / \mathrm{kg}$ ), 0.1\% DMSO or aspirin (50 mg/kg). The mice tail was cut in $3 \mathrm{~mm}$, immersed in normal saline directly and the time of bleeding was recorded until no sign of further bleeding for at least $10 \mathrm{~s}$.

\subsection{Platelet Aggregation}

The directives of the Helsinki Declaration were conformed this study and further approved by the Institutional Review Board of Taipei Medical University (TMU-N201812024). According to our earlier study, the platelet suspensions were prepared from 36 healthy human bloods [14] and mixed with an acid-citrate-dextrose solution $(9: 1, v / v)$. The platelet-rich plasma separated by centrifugation was supplemented with $\mathrm{PGE}_{1}(0.5 \mu \mathrm{M})$ and heparin $(6.4 \mathrm{IU} / \mathrm{mL})$. A $3.5 \mathrm{mg} / \mathrm{mL}$ of BSA and $1 \mathrm{mM}$ of $\mathrm{Ca}^{2+}$ added Tyrode's solution was used to make the final suspensions of washed human platelets.

Platelet aggregation was measured by using a Lumi-Aggregometer (Payton, Scarborough, ON, Canada) through turbidimetric method [14]. Different concentrations of CBN, $0.1 \%$ DMSO, or other substances were preincubated with platelet suspensions $\left(3.6 \times 10^{8}\right.$ cells $\left./ \mathrm{mL}\right)$ for $3 \mathrm{~min}$ before the addition of collagen $(1 \mu \mathrm{g} / \mathrm{mL})$. The incubation was continued further $6 \mathrm{~min}$, and the degree of aggregation was measured as a percentage of the control (Tyrode's solution-treated group) in light-transmission units. A $20 \mu \mathrm{L}$ of a luciferin/luciferase mixture was added $1 \mathrm{~min}$ before the addition of collagen, and the final suspension was used to measure ATP release using Hitachi Spectrometer F-7000 (Tokyo, Japan).

\subsection{Immunoblotting Study}

$\mathrm{CBN}(45$ and $90 \mu \mathrm{M})$ or various reagents were preincubated into washed platelets $\left(1.2 \times 10^{9} \mathrm{cells} / \mathrm{mL}\right)$ for $3 \mathrm{~min}$, before the addition of collagen $(1 \mu \mathrm{g} / \mathrm{mL})$ to trigger platelet activation. After the incubation was terminated, platelets were directly resuspended in $200 \mu \mathrm{L}$ lysis buffer and centrifuged at $5000 \times g$ for $5 \mathrm{~min}$. Supernatants comprising $80 \mu \mathrm{g}$ of protein were electrophoretically separated by $12 \%$ sodium dodecylsulfate polyacrylamide gel electrophoresis (SDS-PAGE). Separated proteins were then transferred through semidry transfer (Bio-Rad, Hercules, CA, USA). The blotting was then blocked using TBST (10 mM Tris-base, $0.01 \%$ Tween 20, and $100 \mathrm{mM} \mathrm{NaCl}$ ) having $5 \% \mathrm{BSA}$ for $1 \mathrm{~h}$. The blocked membranes were incubated with respective primary antibodies (diluted 1:1000 in TBST). The primary antibodies of $p$-I $\kappa \mathrm{B} \alpha, \mathrm{p}-\mathrm{p} 65, \mathrm{I} \kappa \mathrm{B} \alpha, \mathrm{p}-\mathrm{p} 38$ MAPK, $\mathrm{t}-\mathrm{p} 38$ MAPK, p-ERK, t-ERK, p-JNK, t-JNK and $\alpha$-tubulin were detected using anti-phospho-IкB $\alpha$ (Cell Signaling, Cat \#9246), anti-phospho-NF-kB p65 (Cell Signaling, Cat \#3031), anti-IкB $\alpha$ (Cell Signaling, Cat \#4812), anti-phospho-p38 MAPK (Cell Signaling, Cat \#9211), anti-p38 MAPK (Cell Signaling, Cat \#9217), anti-phospho-p44/p42 ERK (Cell Signaling, Cat \#9101), anti-p44/p42 ERK (Cell Signaling, Cat \#9107), 
anti-phospho-c-JNK (Cell Signaling, Cat \#9251), anti-c-JNK (Cell Signaling, Cat \#9252) and anti- $\alpha$-tubulin (Neomarkers, MS-581-p1). And then with HRP-conjugated anti-mouse or anti-rabbit IgG (Amersham, Buckinghamshire, UK; diluted 1:3000 in TBST) for $1 \mathrm{~h}$. The ratios of semiquantitative results obtained by scanning reactive bands and quantified optical density through videodensitometry (Bio-profil; Biolight Windows Application V2000.01; Vilber Lourmat, France).

\subsection{Measurement of Hydroxyl Radicals}

The electron spin resonance (ESR) method was used to measure hydroxyl radicals (HO^) by using a Bruker EMX ESR spectrometer, as described previously [15]. In brief, platelet suspensions $\left(3.6 \times 10^{8}\right.$ cells $\left./ \mathrm{mL}\right)$ were preincubated with CBN $(45$ and $90 \mu \mathrm{M})$ or BAY11-7082 $(8 \mu \mathrm{M})$ for $3 \mathrm{~min}$ before the addition of collagen $(1 \mu \mathrm{g} / \mathrm{mL})$. The incubation was continued for $5 \mathrm{~min}$ before DMPO $(100 \mu \mathrm{M})$ added.

\subsection{Data Analysis}

The results are represented as the mean \pm standard error of the mean (SEM) and are convoyed by the number of observations $(n)$. Differences between mice groups were analyzed using unpaired Student's $t$ tests. Differences among the experimental setups were designed using a one-way analysis of variance (ANOVA). If ANOVA revealed significant differences in the group means, then each group was compared using the Student-Newman-Keuls method. $p<0.05$ was reflected statistically significant.

\section{Results}

\subsection{Effect of CBN in Platelet Plug Formation in Human Whole Blood and Tail Bleeding in Experimental Mice}

Hou et al. [12] reported that $10 \mathrm{mg} / \mathrm{kg}$ CBN treatment markedly prolonged occlusion time in the mesenteric microvessels of mice. In the current study, we further confirmed the effective antithrombotic activity of CBN by using the platelet function analyzer (PFA-100) (Figure 1A), which consists of an instrument and test cartridges in which the process of platelet adhesion and aggregation following a vascular injury is stimulated in vitro. The single-use cartridges consists of a number of integrated parts including a capillary, a sample reservoir and a biochemically active membrane with a central circular aperture (Figure 1A). Citrated whole blood is aspirated from the sample reservoir through the capillary and aperture, which expose platelets to high shear flow conditions (5000-6000/s). The membrane is coated with collagen, a subendothelial protein generally believed to be the initial matrix for platelet attachment. In addition, the membrane is coated with ADP, which is other physiological agonist, along with collagen, are extensively used to trigger platelets in aggregometry testing. The time needed to occlude the aperture is described as closure time (CT). In the present study, the CT of the CADP membrane in whole blood treated with solvent control (0.1\% DMSO) was $81.5 \pm 5.1 \mathrm{~s}(n=8)$; CBN $(90 \mu \mathrm{M})$ significantly prolonged CT $(45 \mu \mathrm{M}, 99.8 \pm 5.6 \mathrm{~s}, n=8 ; p>0.05 ; 90 \mu \mathrm{M}, 114.0 \pm 9.8 \mathrm{~s}, n=8$; $p<0.05)$ compared with the solvent control (Figure 1A).

We evaluated the effects of CBN on bleeding time, since it is a general side effect of antiplatelet drugs when they are using in clinical trials. This effect was also compared with antiplatelet drug aspirin. The bleeding time was $165.4 \pm 15.9 \mathrm{~s}(n=8)$ in the solvent control group (Figure 1B). After $30 \mathrm{~min}$ of intraperitoneal treatment of CBN at 10 and $20 \mathrm{mg} / \mathrm{kg}$, the bleeding time was $180.4 \pm 20.1$ and $191.0 \pm 26.1 \mathrm{~s}(n=8)$, respectively; therefore, the bleeding time was not significantly affected by those doses. In addition, aspirin was administered at $50 \mathrm{mg} / \mathrm{kg}$, and the bleeding time was markedly prolonged after $30 \mathrm{~min}$ from $165.4 \pm 15.9 \mathrm{~s}(0.1 \%$ DMSO-treated group) to $225.7 \pm 6.3 \mathrm{~s}(n=8, p<0.05)$ (Figure 1B). 
A

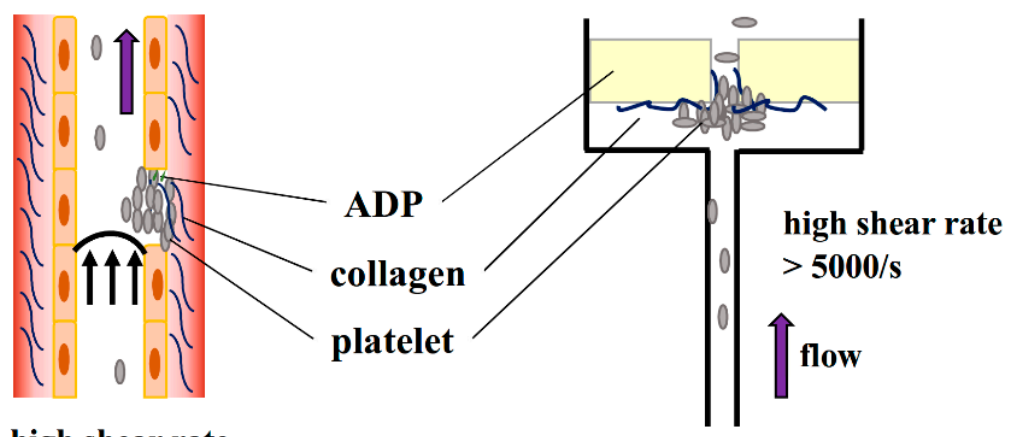

high shear rate

$>5000 / \mathrm{s}$

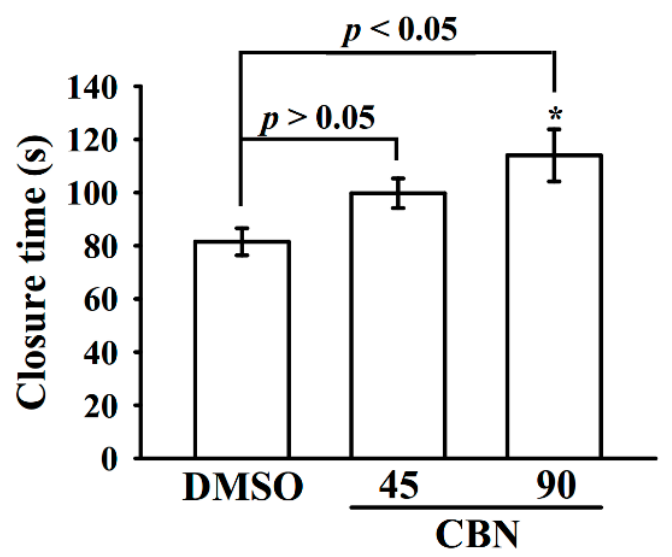

B

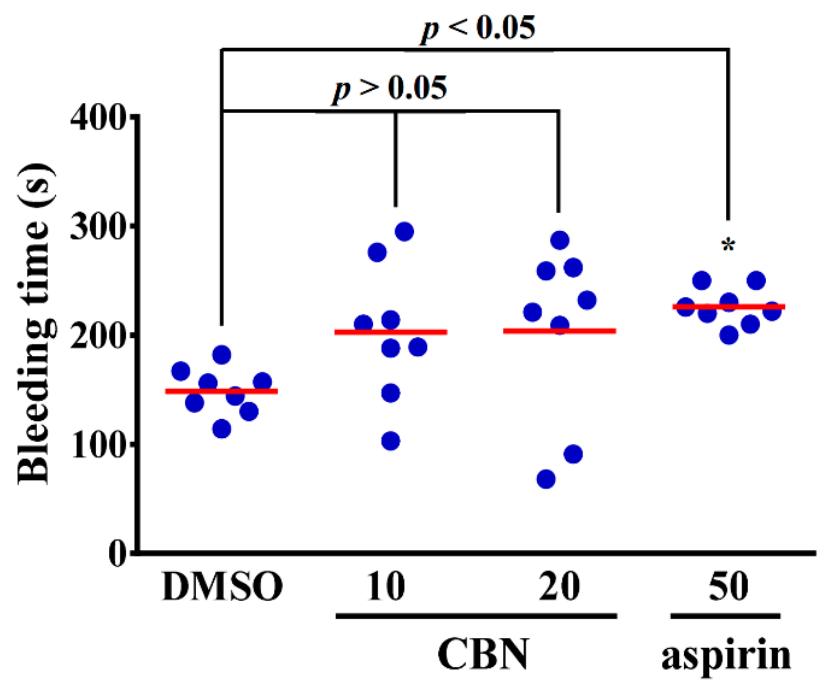

Figure 1. Activity of columbianadin on the closure and bleeding time in mice. (A) High shear flow-induced platelet plug formation in human whole blood was determined by recording the closure time of collagen/ADP (CADP) membrane were preincubated with $0.1 \%$ DMSO, 45 or $90 \mu \mathrm{M}$ columbianadin $(\mathrm{CBN})$ as described in the Materials and Methods. (B) For the animal study, the bleeding time was measured by transection of mouse tails, $30 \mathrm{~min}$ after administering either $0.1 \%$ DMSO, 10 and $20 \mathrm{mg} / \mathrm{kg} \mathrm{CBN}$, or $50 \mathrm{mg} / \mathrm{kg}$ aspirin (positive control to compare with CBN) intraperitoneally. ${ }^{*} p<0.05$, compared with the $0.1 \%$ DMSO-treated group $(n=8)$. 
3.2. Inhibition of Platelet Aggregation and ATP-Release by BAY11-7082, MG-132, and CBN in Collagen-Stimulated Platelets

BAY11-7082 is an IKK inhibitor that possesses several biological activities, including anticancer, neuroprotective, and anti-inflammatory effects [16]. MG-132 belongs to a proteasome inhibitor that blocks the activation of NF- $\kappa B$ by inhibiting I $\mathrm{KB}$ degradation [17]. In the present study, we compared the relative activities of BAY11-7082 and MG-132 in the inhibition of platelet aggregation and determined that in washed human platelets, both BAY11-7082 (4 and $8 \mu \mathrm{M})$ and MG-132 (60 and $120 \mu \mathrm{M})$ inhibited collagen $(1 \mu \mathrm{g} / \mathrm{mL})$-stimulated platelet aggregation in a concentration-dependent manner, similar to CBN (45 and $90 \mu \mathrm{M}$ ) (Figure 2A). BAY11-7082 exhibited a higher potency than MG-132 in on molar basis (Figure 2A). Besides, the release of granular contents (e.g., ATP) is associated with platelet activation, which causes platelet aggregation. As illustrated in Figure 2B, BAY11-7082 also showed higher activity than MG-132 at inhibiting ATP-release stimulated by collagen. Therefore, BAY11-7082 was employed to clarify the role of NF- $\mathrm{KB}$ in CBN-mediated antiplatelet activity.

\subsection{Reduction of NF- $\kappa B$ Signals by $C B N$ in Platelets}

NF- $\mathrm{KB}$ exists as an inactive cytoplasmic complex, composed of heterodimer p50 and p65 subunits that are tightly bound to I $\kappa B$ inhibitory proteins [18]. Figure 3 shows that $I \kappa B \alpha$ and $p 65$ phosphorylation and IкB $\alpha$ protein degradation were significantly increased after stimulation with collagen $(1 \mu \mathrm{g} / \mathrm{mL})$ in human platelets. CBN (45 and $90 \mu \mathrm{M}$ ) reduced I $\kappa \mathrm{B} \alpha$ and p65 phosphorylation (Figure $3 \mathrm{~A}, \mathrm{~B}$ ) and reversed I $\mathrm{K} B \alpha$ degradation (Figure $3 \mathrm{C}$ ) caused by collagen stimulation. Assembled data of Figure 3 are displayed in right panels. These results suggest that the inhibition of NF- $\mathrm{KB}$ signals may play a critical role in CBN-mediated antiplatelet activity.

\subsection{Connection Between NF- $\kappa B$ Signaling and MAPK Phosphorylation}

MAPK families play central roles in complex cellular programs, such as platelet activation, cell proliferation, differentiation and apoptosis [19]. At least three major MAPK families have been well characterized: ERK1/2, JNK1/2, and p38 MAPK. We employed various inhibitors for additional investigation on cellular signaling between NF-KB and MAPKs. First, pretreatment with CBN $(90 \mu \mathrm{M})$ reduced the phosphorylation of both ERK1/2 and JNK1/2, but not p38 MAPK, after stimulation with collagen (Figure 4A), which accords with previous findings [12]. Furthermore, both BAY11-7082 $(8 \mu \mathrm{M})$ and CBN $(90 \mu \mathrm{M})$ almost inhibited collagen-stimulated ERK1/2 and JNK1/2 phosphorylation (Figure 4B,C). Pretreatment with PD98059 (10 $\mu$ M; ERK phosphorylation inhibitor) and SP600125 $(10 \mu \mathrm{M}$; JNK phosphorylation inhibitor) reduced ERK1/2 and JNK1/2 phosphorylation, respectively (Figure 4B,C). Moreover, CBN $(90 \mu \mathrm{M})$ and BAY11-7082 $(8 \mu \mathrm{M})$ reduced collagen-stimulated IKB $\alpha$ phosphorylation (Figure 5A,B). Interestingly, PD98059 (10 $\mu \mathrm{M}$; Figure 5A) but not SP600125 (10 $\mu \mathrm{M}$; Figure $5 B$ ) inhibited I $\kappa B \alpha$ phosphorylation, suggesting that NF- $k B$ and ERK1/2 activate each other, and JNK1/2 phosphorylation seems to be regulated by NF- $\mathrm{KB}$ in collagen-activated platelets. 
A
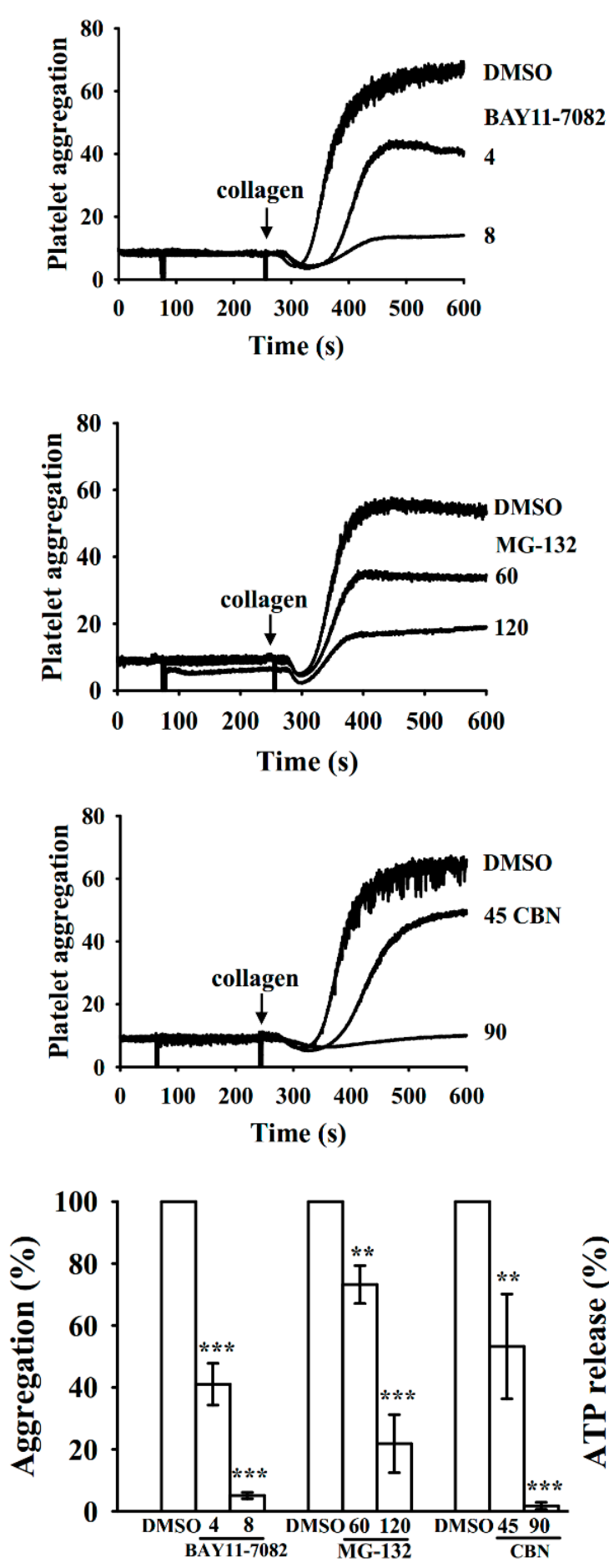

\section{B}
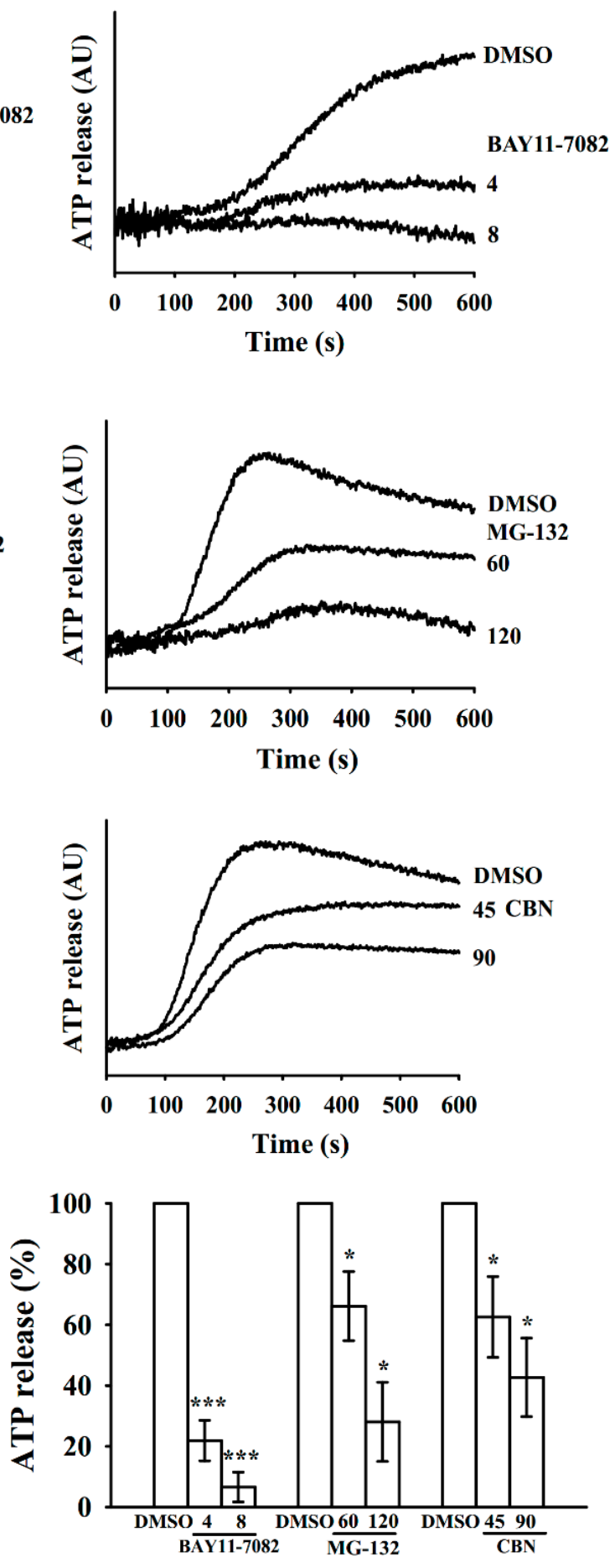

Figure 2. Inhibitory activity of BAY11-7082, MG-132, and columbianadin in human platelet aggregation and ATP-release. Washed human platelets were preincubated with BAY11-7082 (IKK inhibitor; 4 and $8 \mu \mathrm{M}$ ), MG-132 (proteasome inhibitor; 60 and $120 \mu \mathrm{M}$ ), and CBN (45 and $90 \mu \mathrm{M}$ ), followed by the addition of collagen $(1 \mu \mathrm{g} / \mathrm{mL})$ to trigger (A) platelet aggregation and (B) ATP-release reaction (AU; arbitrary unit). The corresponding statistical data are illustrated in the low panel of figures. ${ }^{*} p<0.05$, ** $p<0.01$ and ${ }^{* * *} p<0.001$, compared with the $0.1 \%$ DMSO-treated group $(n=4)$. 

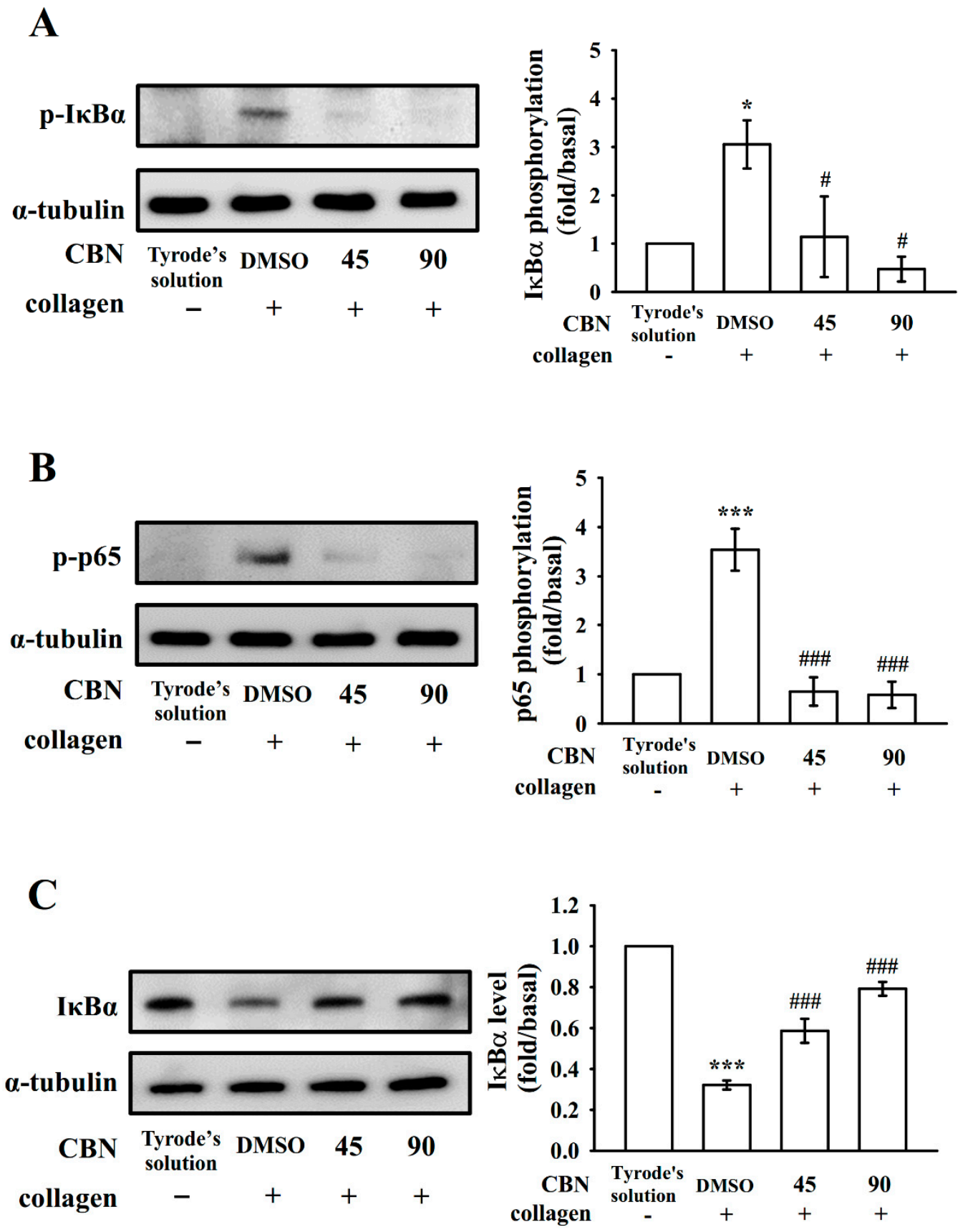

Figure 3. Inhibition of NF- $\kappa B$ activation by columbianadin in washed human platelets. (A) IкB $\alpha$, (B) p65 phosphorylation, and (C) I $\kappa \mathrm{B} \alpha$ protein degradation. ${ }^{*} p<0.05$ and ${ }^{* * *} p<0.001$, compared with resting platelets; \# $p<0.05$ and \#\#\# $p<0.001$, compared with the $0.1 \%$ DMSO-treated group $(n=4)$. 


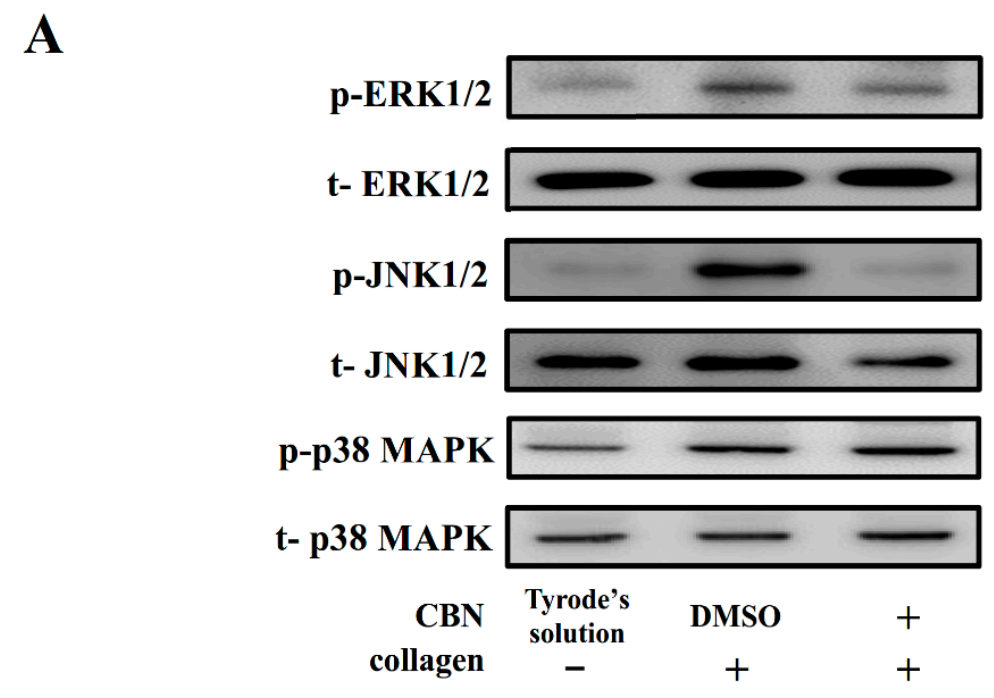

B
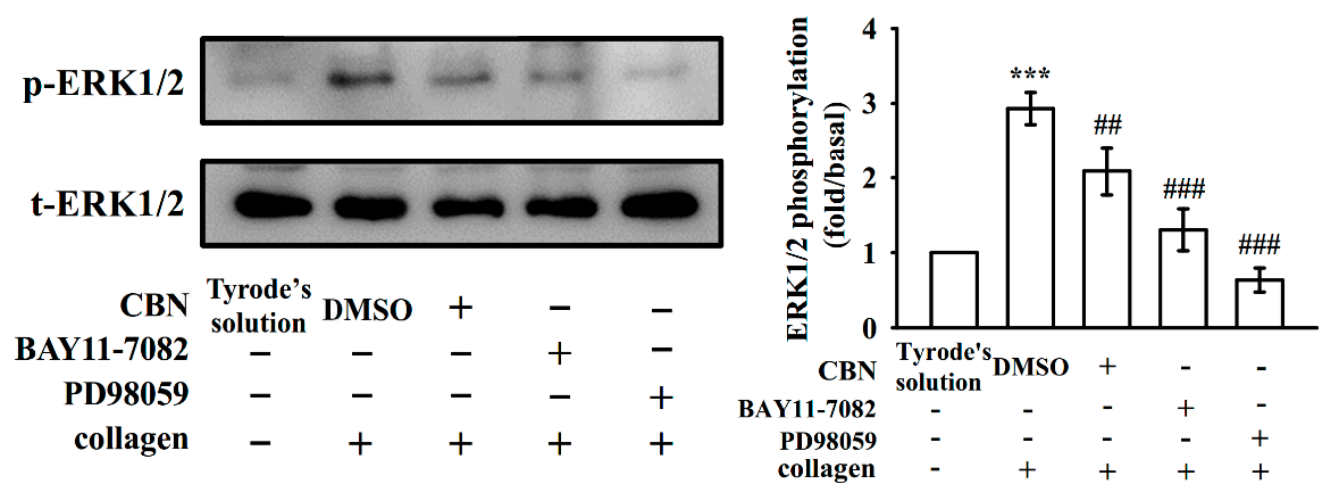

C
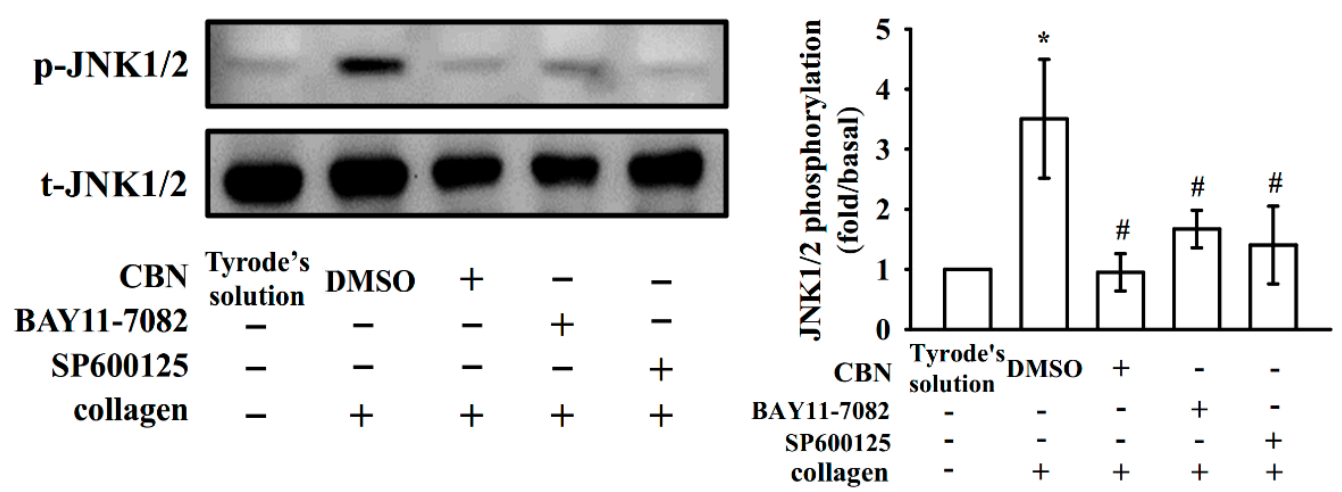

Figure 4. Effects of BAY11-7082 on ERK1/2 and JNK1/2 phosphorylation in collagen-activated washed human platelets. Washed platelets were preincubated with 0.1\% DMSO, CBN (90 $\mu \mathrm{M})$, BAY11-7082 $(8 \mu \mathrm{M})$, PD98059 (ERK inhibitor; $10 \mu \mathrm{M}$ ) or SP600125 (JNK inhibitor; $10 \mu \mathrm{M}$ ) and then treated with collagen $(1 \mu \mathrm{g} / \mathrm{mL})$ to induce platelet activation. The levels of (A) ERK1/2, JNK1/2, and p38 MAPK phosphorylation were detected in the subcellular extracts of the collected platelets. The matching statistical data of $(\mathbf{B}, \mathbf{C})$ are displayed on the right panel of each figure. All phosphorylated proteins were normalized to the total proteins. ${ }^{*} p<0.05$ and ${ }^{* * *} p<0.001$, compared with resting platelets; $\# p<0.05$, \#\# $p<0.01$, and \#\#\# $p<0.001$, compared with the $0.1 \%$ DMSO-treated group $(n=4)$. 


\section{A}
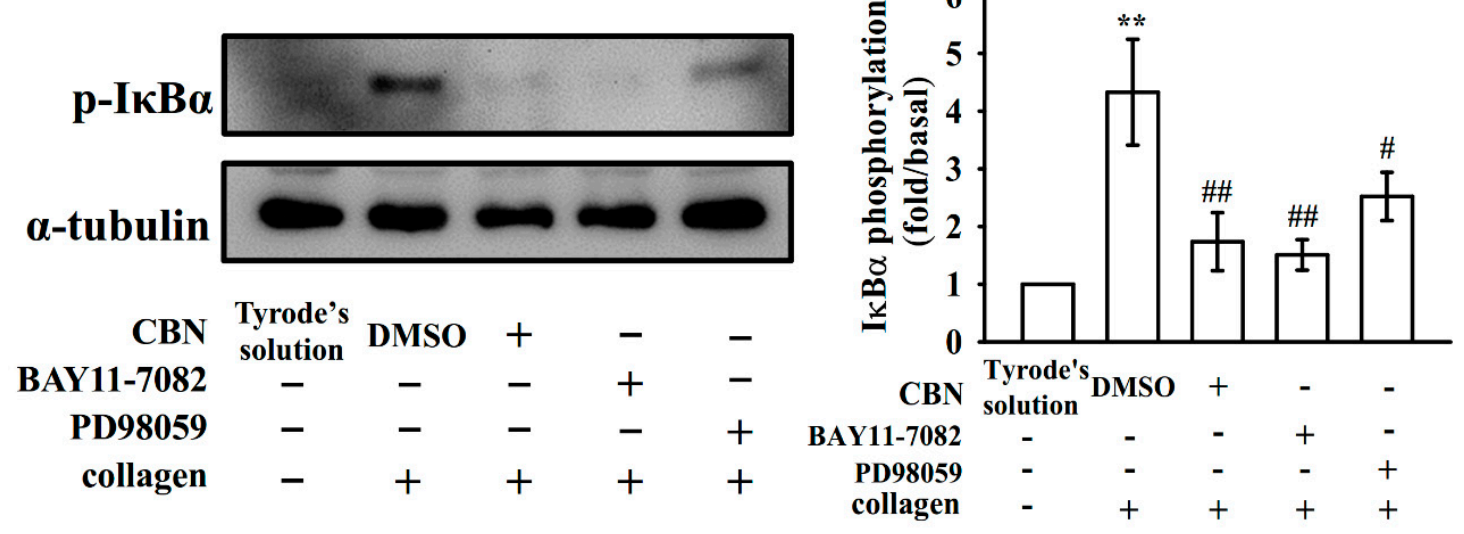

B

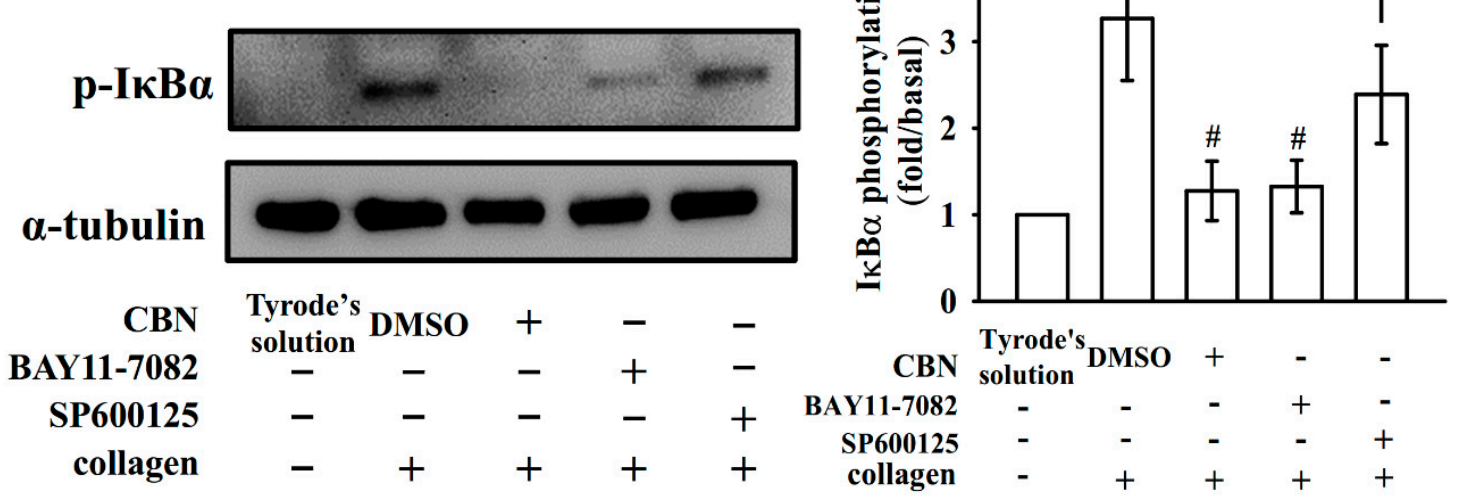

Figure 5. Effect of the PD98059 (A) or SP600125 (B) on I $\kappa \mathrm{B} \alpha$ protein phosphorylation in washed platelets. ${ }^{*} p<0.05$ and ${ }^{* *} p<0.01$, compared with resting platelets; \# $p<0.05$, and \#\# $p<0.01$, compared with the $0.1 \%$ DMSO-treated group $(n=4)$.

\subsection{Effect of CBN and BAY11-7082 on HO• Formation}

Reactive oxygen species (ROS) generated from platelet activation may further increase platelet reactivity during thrombus formation. However, the controlling pathways of ROS, particularly $\mathrm{HO}^{\bullet}$, in platelet activation remain unclear. As illustrated in Figure 6, a classic ESR signal of $\mathrm{HO}^{\bullet}$ was triggered by collagen $(1 \mu \mathrm{g} / \mathrm{mL}$ ), which was less in resting platelets (Figure $6 \mathrm{~A}, \mathrm{~B}$ ); $90 \mu \mathrm{M}$ but not $45 \mu \mathrm{M}$ of CBN obviously reduced $\mathrm{HO}^{\bullet}$ formation (Figure 6C,D), whereas BAY11-7082 (8 $\mu \mathrm{M}$ ) had no noteworthy effect under the same condition (Figure 6E). 

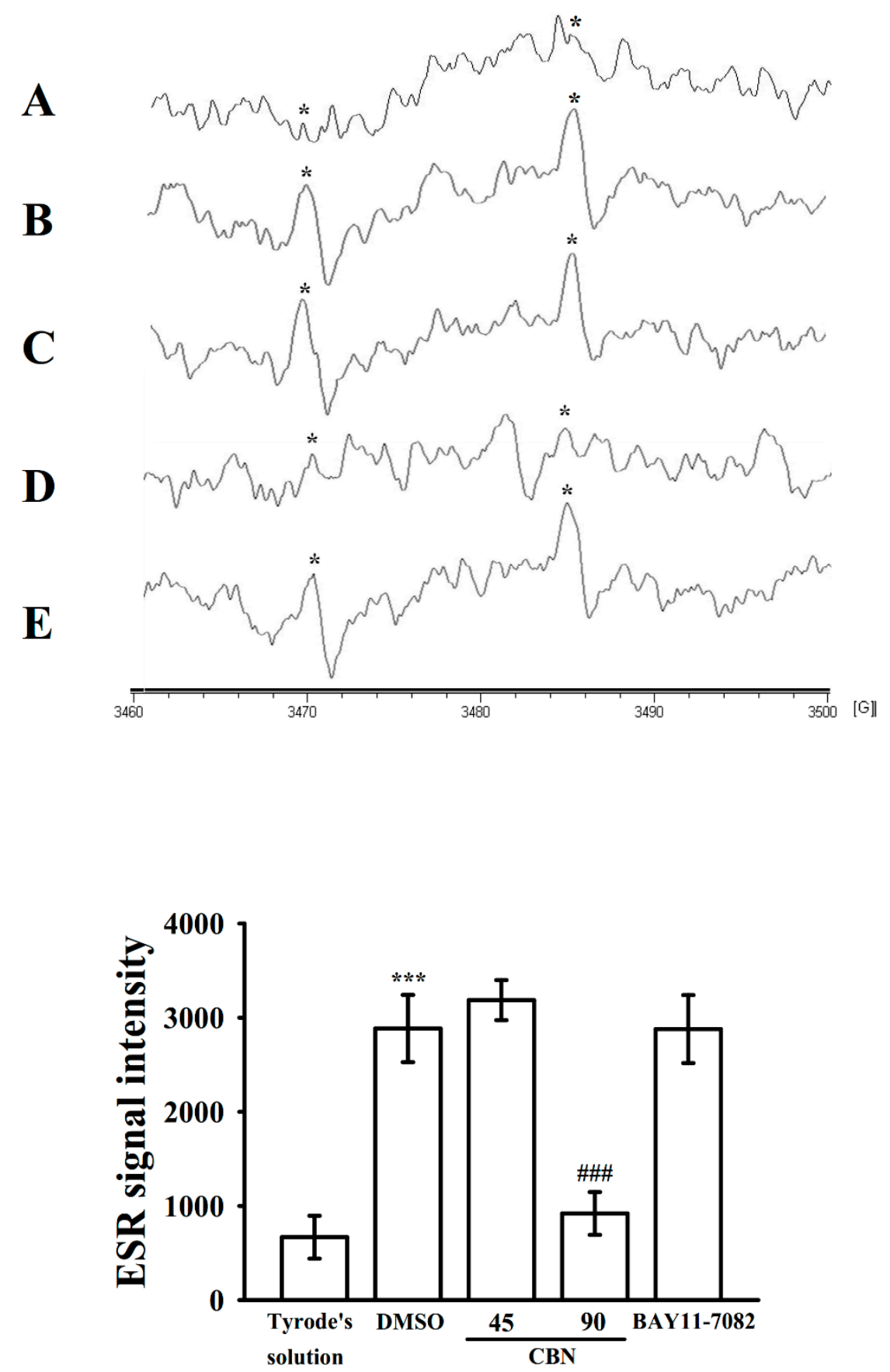

Figure 6. Activity of $\mathrm{CBN}$ on $\mathrm{HO}^{\bullet}$ formation in human platelets. For ESR study, washed platelets were incubated with (A) Tyrode's solution (resting group), (B) 0.1\% DMSO, CBN; (C) $45 \mu \mathrm{M}$; (D) $90 \mu \mathrm{M}$ ), or (E) BAY11-7082 $(8 \mu \mathrm{M})$, followed by the addition of collagen $(1 \mu \mathrm{g} / \mathrm{mL})$ to trigger $\mathrm{HO}^{\bullet}\left({ }^{*}\right)$ formation. *** $p<0.001$, compared with resting platelets; \#\#\# $p<0.001$, compared with the $0.1 \%$ DMSO-treated group $(n=4)$.

\section{Discussion}

Columbianadin is a natural coumarin derivative from Angelica decursiva (Umbelliferae) have various biological activities, including anti-inflammatory and anticancer [10]. Collagens from the subendothelial matrix activates platelet adhesion and aggregation at the site of vascular endothelial cell injury, which is then, induced arterial thrombus formation. Previous study [12] demonstrated that CBN significantly reduced the mortality of mice those were subjected to ADP-induced acute 
pulmonary thromboembolism. Platelet adhere to collagen depended on flow conditions, and inactive platelets were unable to adhere to the CADP membrane under flow conditions. In the present study, we confirmed that CBN markedly prolonged closure time (CT) in whole blood by using the PFA-100 instrument. Therefore, CBN may act as potent agent in the inhibition of human platelet activation and suggesting that it can have therapeutic or prophylactic applications. Moreover, the effect of CBN on bleeding time was examined by using the tail transection model in mice. Aspirin is the highly recommended antiplatelet drug for the prevention or treatment of cerebro-cardiovascular diseases. CBN-treated mice were not significantly prolongation of bleeding time, whereas aspirin-treated mice caused the prolongation under the same condition. Therefore, CBN may signify an active coumarin derivative for treating thromboembolic disorders without causing the side effect of bleeding.

In this study, CBN inhibited collagen-stimulated NF- $\kappa B$ activation in human platelets, indicating that NF- $\mathrm{KB}$ signals play a crucial role in $\mathrm{CBN}$-mediated antiplatelet activity. The function of NF- $k B$ in nucleated cells has been studied extensively. Diverse stimuli, including bacterial infection, cytokines, and free radicals can induce NF- $\mathrm{KB}$ activation. NF- $\mathrm{KB}$ regulates genes involved in cell survival, cell proliferation and inflammation responses among others [18]. Therefore, NF- $\kappa B$ is an ideal targeting transcription factor for therapeutic interventions against inflammatory diseases and cancer. Several studies have demonstrated that platelets express several transcription factors [20,21], suggesting that these transcription factors have a nongenomic function in platelets. However, whether NF- $\mathrm{KB}$ is practically present in a novel manner in platelets, unrelated to transcriptional regulation, remains unknown. NF- $\mathrm{kB}$ was reported to be involved in thromboxane $\mathrm{A}_{2}\left(\mathrm{TxA}_{2}\right)$ formation, P-selectin expression, fibrinogen adhesion in platelets [22]. In the current study, BAY11-7082 inhibited platelet aggregation, ATP-release reaction and phosphorylation of $\operatorname{I} \mathrm{B} \alpha$, which definitively demonstrated that NF- $\mathrm{kB}$ is involved in platelet activation through various signal pathways. So far, we are not clear whether $\mathrm{CBN}$ directly regulates NF- $\mathrm{KB}$, or through inhibition of its upstream regulator, hence it needs to be investigated in the future. Liu et al. [23] were the first to demonstrate the expression of NF- $\mathrm{KB}$ in platelets in 2002, revealing that thrombin-induced platelet activation triggers the degradation of $I \kappa B \alpha$ following its serine 32 residue phosphorylation. [23].

As described previously [11], CBN inhibits platelet activation through interfering in integrin $\alpha_{\mathrm{IIb}} \beta_{3}$ inside-out signals. Salanova et al. [24] reported that platelet integrin $\alpha_{\text {IIb }} \beta_{3}$ colocalized with integrin $\beta_{2}$ and cooperated in NF-KB activation, our results accord with their findings. MAPKs are serine/threonine protein kinases consist of of three major subgroups, p38 MAPK, ERK1/2, and JNK1/2, which occurred in platelets and activated by various agonists [25]. Our findings demonstrated that BAY11-7082 attenuates collagen induced JNK1/2 and ERK1/2 phosphorylation in human platelets. Similarly, Kauskot et al. [26] found the involvement of JNK1 in ADP-dependent collagen-induced platelet aggregation. They also demonstrated that glycoprotein (GP)Ib-von Willebrand factor (vWF) interaction triggers integrin $\alpha_{\mathrm{IIb}} \beta_{3}$ activation in a JNK1-dependent manner during rolling and adhesion of platelets to vWF. Several studies with different experimental setups have indicated that ERK2 is also involved in platelet aggregation. Roger et al. [27] and Falker et al. [28] have independently reported the involvement of ERK2 in platelet aggregation. They demonstrated that collagen- or thrombin-induced ERK2 activation depends on $\mathrm{TxA}_{2}$ formation and ADP/ATP release reaction. Furthermore, Toth-Zsamboki et al. [29] have indicated that ERK2 affects platelet secretion.

A partial amount of hydrogen peroxide formed by platelets is converted into $\mathrm{HO}^{\bullet}$, which are involved in the initial phase of platelet activation [15]. The ESR study revealed that BAY11-7082 did not scavenge $\mathrm{HO}^{\bullet}$ in activated platelets, indicating that platelet $\mathrm{HO} \mathrm{O}^{\bullet}$ formation does not seem to be regulated by NF- $\mathrm{kB}$ signal, and which may be played an upstream regulator for NF- $\mathrm{kB}$ signal. However, we do not rule out the possibility that other unknown signals are involved in the NF-kB-mediated inhibition of platelet activation by CBN. 


\section{Conclusions}

This study presents a distinctive inhibitory pathway of NF-KB-mediated MAPK activation by $C B N$ in human platelets and corroborates findings that $C B N$ has potent activity and thus should be investigated as a prophylactic or clinical therapy for cardiovascular diseases (Figure 7). Platelet activation is involved in inflammation; thus, blocking platelet function by inhibiting NF- $\mathrm{KB}$ signal could also be considered for treating various inflammatory diseases.

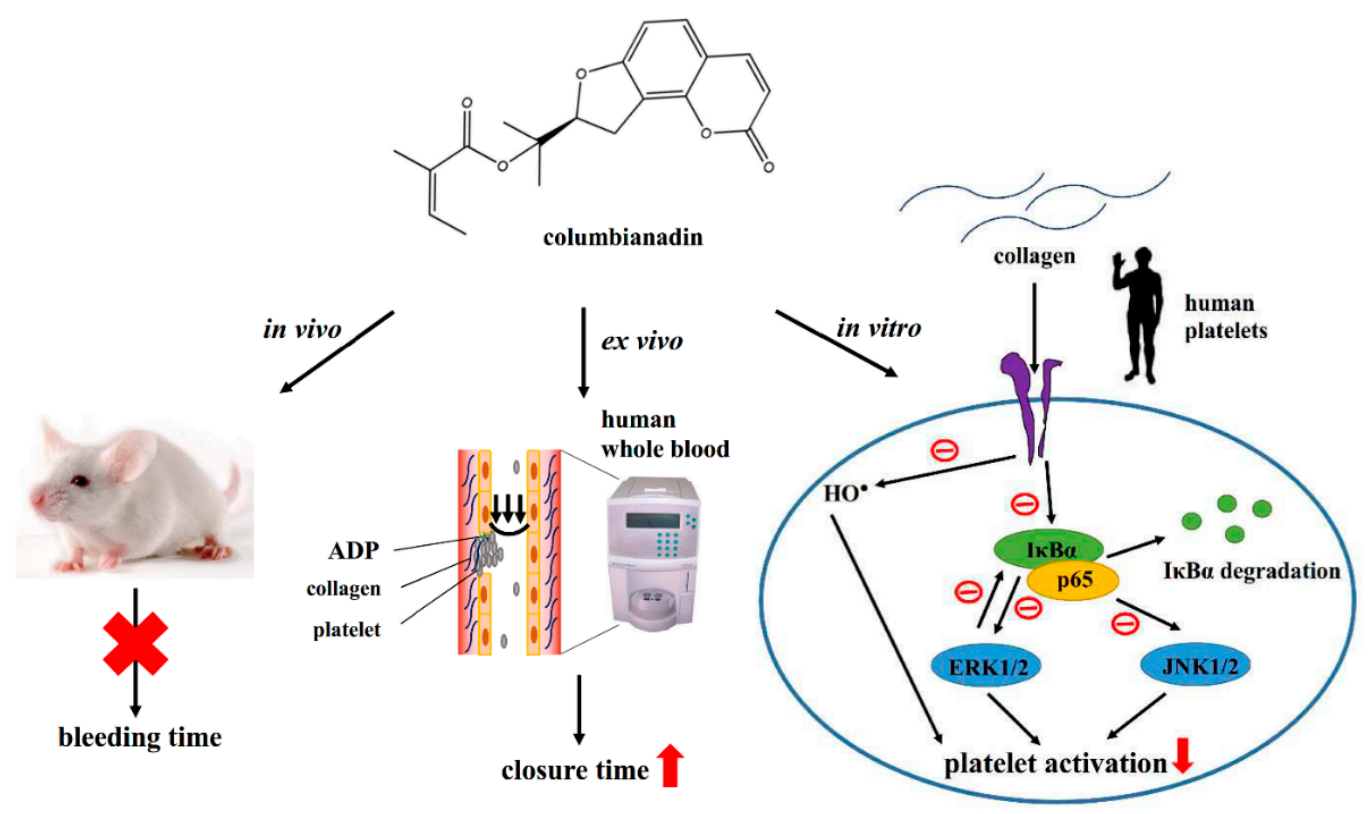

Figure 7. Hypothetical scheme of inhibitory signaling by columbianadin in human platelet activation and tail bleeding in mice. Columbianadin ( $\mathrm{CBN}$ ) inhibits hydroxyl radical (HO•) formation and NF- $\mathrm{kB}$ activation including I $\mathrm{K} \mathrm{B} \alpha$ phosphorylation and degradation, and $\mathrm{p} 65$ phosphorylation, which followed by the inhibition of MAPK activation (ERK1/2 and JNK1/2 phosphorylation), and finally inhibits platelet activation stimulated by collagen in human platelets. Moreover, the closure time of the platelet plug formation in human whole blood significantly prolonged by CBN, whereas CBN did not pointedly prolong the bleeding time in experimental mice.

Author Contributions: C.-W.H., C.-H.Y. and J.-R.S. performed research and wrote the manuscript; C.-H.H., C.-L.T., W.-C.H. and T.-Y.C. performed research and the partial experiments; T.J. and P.S.B. performed the partial experiments and analyzed data; Y.C. conceived of the study and designed research. All authors read and approved the final manuscript.

Funding: This work was supported by grants from the Ministry of Science and Technology of Taiwan (MOST107-2320-B-038-035-MY2 and MOST108-2320-B-038-031-MY3), Shin Kong Wu Ho-Su Memorial Hospital (2018SKHADR023), and Shin Kong Wu Ho-Su Memorial Hospital-Taipei Medical University (SKH-TMU-106-06), and Taipei Medical University (DP2-109-21121-01-N-08-03).

Acknowledgments: The Wallace Academic Editing acknowledged for editing this manuscript.

Conflicts of Interest: We have no conflict of interest.

\section{References}

1. Beckman, M.G.; Hooper, W.C.; Critchley, S.E.; Ortel, T.L. Venous thromboembolism: A public health concern. Am. J. Prev. Med. 2010, 38, S495-S501.

2. George, J.N. Platelets. Lancet 2000, 355, 1531-1539. [PubMed]

3. Xu, X.R.; Zhang, D.; Oswald, B.E.; Carrim, N.; Wang, X.; Hou, Y.; Zhang, Q.; Lavalle, C.; McKeown, T.; Marshall, A.H.; et al. Platelets are versatile cells: New discoveries in hemostasis, thrombosis, immune responses, tumor metastasis and beyond. Crit. Rev. Clin. Lab. Sci. 2016, 53, 409-430. [PubMed] 
4. Pikarsky, E.; Porat, R.M.; Stein, I.; Abramovitch, R.; Amit, S.; Kasem, S. NF-kappaB functions as a tumour promoter in inflammation-associated cancer. Nature 2004, 431, 461-466.

5. Kojok, K.; El-Kadiry, A.E.; Merhi, Y. Role of NF-kB in Platelet Function. Int. J. Mol. Sci. 2019, $20,4185$.

6. Lee, H.S.; Kim, S.D.; Lee, W.M.; Endale, M.; Kamruzzaman, S.M.; Oh, W.J.; Cho, J.Y.; Kim, S.K.; Cho, H.J.; Park, H.J.; et al. A noble function of BAY 11-7082: Inhibition of platelet aggregation mediated by an elevated cAMP-induced VASP, and decreased ERK2/JNK1 phosphorylations. Eur. J. Pharmacol. 2010, 627, 85-91. [PubMed]

7. Fuentes, E.; Rojas, A.; Palomo, I. NF-кB signaling pathway as target for antiplatelet activity. Blood Rev. 2016, 30, 309-315. [PubMed]

8. Rivadeneyra, L.; Carestia, A.; Etulain, J.; Pozner, R.G.; Fondevila, C.; Negrotto, S.; Schattner, M. Regulation of platelet responses triggered by Toll-like receptor 2 and 4 ligands is another non-genomic role of nuclear factor-kappaB. Thromb. Res. 2014, 133, 235-243.

9. Kang, J.I.; Hong, J.Y.; Choi, J.S.; Lee, S.K. Columbianadin inhibits cell proliferation by inducing apoptosis and necroptosis in HCT116 colon cancer cells. Biomol. Ther. 2016, 24, 320-327.

10. Zhang, C.; Hsu, A.C.; Pan, H.; Gu, Y.; Zuo, X.; Dong, B.; Wang, Z.; Zheng, J.; Lu, J.; Zheng, R.; et al. Columbianadin Suppresses Lipopolysaccharide (LPS)-Induced Inflammation and Apoptosis through the NOD1 Pathway. Molecules 2019, 24, 549.

11. Lim, H.J.; Lee, J.H.; Choi, J.S.; Lee, S.K.; Kim, Y.S.; Kim, H.P. Inhibition of airway inflammation by the roots of Angelica decursiva and its constituent, columbianadin. J. Ethnopharmacol. 2014, 155, 1353-1361.

12. Hou, S.M.; Hsia, C.W.; Tsai, C.L.; Hsia, C.H.; Jayakumar, T.; Velusamy, M.; Sheu, J.R. Modulation of human platelet activation and in vivo vascular thrombosis by columbianadin: Regulation by integrin $\alpha \operatorname{Ilb} \beta 3$ inside-out but not outside-in signals. J. Biomed. Sci. 2020, 27, 60. [PubMed]

13. Jilma, B. Platelet function analyzer (PFA-100): A tool to quantify congenital or acquired platelet dysfunction. J. Lab. Clin. Med. 2001, 138, 152-163.

14. Chen, W.F.; Lee, J.J.; Chang, C.C.; Lin, K.H.; Wang, S.H.; Sheu, J.R. Platelet protease-activated receptor (PAR)4, but not PAR1, associated with neutral sphingomyelinase responsible for thrombin-stimulated ceramide-NF-kB signaling in human platelets. Haematologica 2013, 98, 793-801.

15. Chou, D.S.; Hsiao, G.; Shen, M.Y.; Tsai, Y.J.; Chen, T.F.; Sheu, J.R. ESR spin trapping of a carbon-centered free radical from agonist-stimulated human platelets. Free Radic. Biol. Med. 2005, 39, 237-248. [PubMed]

16. Lin, Y.; Bai, L.; Chen, W.; Xu, S. The NF-kappaB activation pathways, emerging molecular targets for cancer prevention and therapy. Expert Opin. Ther. Targets 2010, 14, 45-55.

17. Lee, D.H.; Goldberg, A.L. Proteasome inhibitors: Valuable new tools for cell biologists. Trends Cell Biol. 1998, 8, 397-403.

18. Ghosh, S.; Hayden, M.S. New regulators of NF-kappaB in inflammation. Nat. Rev. Immunol. 2008, 8, 837-848.

19. Cargnello, M.; Roux, P.P. Activation and function of the MAPKs and their substrates, the MAPK-activated protein kinases. Microbiol. Mol. Biol. Rev. 2011, 75, 50-83.

20. Ali, F.Y.; Davidson, S.J.; Moraes, L.A.; Traves, S.L.; Paul-Clark, M.; Bishop-Bailey, D.; Warner, T.D.; Mitchell, J.A. Role of nuclear receptor signaling in platelets: Antithrombotic effects of PPARbeta. FASEB J. 2006, 20, 326-328. [PubMed]

21. Moraes, L.A.; Paul-Clark, M.J.; Rickman, A.; Flower, R.J.; Goulding, N.J.; Perretti, M. Ligand-specific glucocorticoid receptor activation in human platelets. Blood 2005, 106, 4167-4175. [PubMed]

22. Malaver, E.; Romaniuk, M.A.; D'Atri, L.P.; Pozner, R.G.; Negrotto, S.; Benzadon, R.; Schattner, M. NF-kappaB inhibitors impair platelet activation responses. J. Thromb. Haemost. 2009, 7, 1333-1343.

23. Liu, F.; Morris, S.; Epps, J.; Carroll, R. Demonstration of an activation regulated NF-kappaB/I-kappaBalpha complex in human platelets. Thromb. Res. 2002, 106, 199-203. [PubMed]

24. Salanova, B.; Choi, M.; Rolle, S.; Wellner, M.; Luft, F.C.; Kettritz, R. Beta2-integrins and acquired glycoprotein $\mathrm{IIb} / \mathrm{IIIa}$ (GPIIb/IIIa) receptors cooperate in NF-kappaB activation of human neutrophils. J. Biol. Chem. 2007, 282, 27960-27969. [PubMed]

25. Bugaud, F.; Nadal-Wollbold, F.; Levy-Toledano, S.; Rosa, J.P.; Bryckaert, M. Regulation of c-jun-NH2 terminal kinase and extracellular-signal regulated kinase in human platelets. Blood 1999, 94, 3800-3805. [PubMed]

26. Kauskot, A.; Adam, F.; Mazharian, A.; Ajzenberg, N.; Berrou, E.; Bonnefoy, A.; Rosa, J.P.; Hoylaerts, M.F.; Bryckaert, M. Involvement of the mitogen-activated protein kinase c-Jun NH2-terminal kinase 1 in thrombus formation. J. Biol. Chem. 2007, 282, 31990-31999. [PubMed] 
27. Roger, S.; Pawlowski, M.; Habib, A.; Jandrot-Perrus, M.; Rosa, J.P.; Bryckaert, M. Costimulation of the Gi-coupled ADP receptor and the Gq-coupled TXA2 receptor is required for ERK2 activation in collagen-induced platelet aggregation. FEBS. Lett. 2004, 556, 227-235.

28. Falker, K.; Lange, D.; Presek, P. ADP secretion and subsequent P2Y12 receptor signalling play a crucial role in thrombin-induced ERK2 activation in human platelets. Thromb. Haemost. 2004, 92, 114-123.

29. Toth-Zsamboki, E.; Oury, C.; Cornelissen, H.; De Vos, R.; Vermylen, J.; Hoylaerts, M.F. P2X1-mediated ERK2 activation amplifies the collagen-induced platelet secretion by enhancing myosin light chain kinase activation. J. Biol. Chem. 2003, 278, 46661-46667.

Publisher's Note: MDPI stays neutral with regard to jurisdictional claims in published maps and institutional affiliations.

(C) 2020 by the authors. Licensee MDPI, Basel, Switzerland. This article is an open access article distributed under the terms and conditions of the Creative Commons Attribution (CC BY) license (http://creativecommons.org/licenses/by/4.0/). 